\title{
Direct Numerical Simulation and Theory of a Wall-Bounded Flow with Zero Skin Friction
}

\author{
G. N. Coleman 1 (1) -S. Pirozzoli ${ }^{2} \cdot$ M. Quadrio ${ }^{3}$. \\ P. R. Spalart ${ }^{4}$
}

Received: 2 March 2017 / Accepted: 8 July 2017

(C) Springer Science+Business Media Dordrecht (outside the USA) 2017

\begin{abstract}
We study turbulent plane Couette-Poiseuille (CP) flows in which the conditions (relative wall velocity $\Delta U_{w} \equiv 2 U_{w}$, pressure gradient $\mathrm{d} P / \mathrm{d} x$ and viscosity $v$ ) are adjusted to produce zero mean skin friction on one of the walls, denoted by APG for adverse pressure gradient. The other wall, FPG for favorable pressure gradient, provides the friction velocity $u_{\tau}$, and $h$ is the half-height of the channel. This leads to a one-parameter family of one-dimensional flows of varying Reynolds number $\operatorname{Re} \equiv U_{w} h / v$. We apply three codes, and cover three Reynolds numbers stepping by a factor of two each time. The agreement between codes is very good, and the Reynolds-number range is sizable. The theoretical questions revolve around Reynolds-number independence in both the core region (free of local viscous effects) and the two wall regions. The core region follows Townsend's hypothesis of universal behavior for the velocity and shear stress, when they are normalized with $u_{\tau}$ and $h$; on the other hand universality is not observed for all the Reynolds stresses, any more than it is in Poiseuille flow or boundary layers. The FPG wall region obeys the classical law of the wall, again for velocity and shear stress. For the APG wall region, Stratford
\end{abstract}

\author{
G. N. Coleman \\ g.n.coleman@nasa.gov \\ S. Pirozzoli \\ sergio.pirozzoli@uniroma1.it \\ M. Quadrio \\ maurizio.quadrio@polimi.it \\ P. R. Spalart \\ philippe.r.spalart@boeing.com \\ 1 NASA Langley Research Center, Hampton, VA 23681, USA \\ 2 Sapienza Università di Roma, Via Eudossiana 18, 00184 Roma, Italy \\ 3 Politecnico di Milano, Via La Masa 34, 20156 Milano, Italy \\ 4 Boeing Commercial Airplanes, P.O. Box 3707, Seattle, WA 98124, USA
}


conjectured universal behavior when normalized with the pressure gradient, leading to a square-root law for the velocity. The literature, also covering other flows with zero skin friction, is ambiguous. Our results are very consistent with both of Stratford's conjectures, suggesting that at least in this idealized flow turbulence theory is successful like it was for the classical logarithmic law of the wall. We appear to know the constants of the law within a $10 \%$ bracket. On the other hand, that again does not extend to Reynolds stresses other than the shear stress, but these stresses are passive in the momentum equation.

Keywords Turbulence $\cdot$ Simulation $\cdot$ Couette-Poiseuille flow

\section{Introduction}

The aim of this study is to test two theoretical conjectures applied to the zero-skin-friction $\mathrm{CP}$ flow, which we will name CP0. The first conjecture is that the velocity profile near the frictionless wall is universal (independent of the flow Reynolds number Re) when velocity and wall distance are both normalized by the pressure gradient and the kinematic viscosity $v$, so that

$$
U^{-}=\mathcal{F}\left(y^{-}\right),
$$

where $U^{-} \equiv U / u_{p}, y^{-} \equiv y_{w} u_{p} / v, y_{w}$ is the wall-normal distance from the frictionless wall, $u_{p}^{3} \equiv \nu \mathrm{d}(P / \rho) / \mathrm{d} x$ and $\rho$ is the (constant) fluid density. The second conjecture is that for large $y^{-}$, allowed by high enough Re, the velocity profile will include a square-root layer, with (1) taking the form

$$
U^{-}=B \sqrt{y^{-}}+C,
$$

where $B$ and $C$ are nondimensional universal constants. These conjectures, which we shall refer to respectively as (I) and (II), come from reasoning, first proposed by Stratford [12], equivalent to that that produce the classical Law of the Wall and logarithmic layer; the skin friction being zero, the normalization is built on the pressure gradient instead. Dimensional analysis then directly leads to the conjectures. There have been attempts to combine $u_{\tau}$ and $u_{p}$ at walls that have both skin friction and pressure gradient, but they lack rigor in our opinion, and we have no plans of that sort. We focus on the pure zero-skin-friction situation.

Although CP0 flow arguably provides the best chance of success for conjectures (I) and (II), its study to date has yet to provide clear conclusions. Schlichting and Gersten [10] cite experiments (not in the $\mathrm{CP} 0$ flow) for which the 'universal constants' range as widely as $2.5<B<4.9$ and $-3.2<C<2.2$, while the direct numerical simulation (DNS) of CP0 flow (Coleman \& Spalart [2]; hereinafter CS15) revealed significant differences with the earlier DNS of Pirozzoli et al. [9] (henceforth PBO11). Although both appear to support the $\sqrt{y_{w}}$ behavior, PBO11 yields $(B, C)=(3.6,-2.65)$ while CS15 suggests $(2.2,-2.05)$. The primary objective of this joint paper is to resolve this discrepancy, via a series of new DNS, and thereby provide unambiguous evidence either for or against conjectures (I) and (II).

In addition to being a testbed for the zero-skin-friction scaling, this flow also satisfies the conditions assumed by Townsend [13] in his derivation of Reynolds-number similarity for the mean velocity profile $U(y)$ in the core of a parallel wall-bounded turbulent shear flow (see his $\S 5.3$ ), which in the present context implies

$$
\frac{U(y)-U_{c}}{u_{\tau}}=\mathcal{F}\left(\frac{y}{h}\right)
$$


where $\tau_{w} \equiv \rho u_{\tau}^{2}$ is the nonzero wall-shear stress (i.e., on the FPG wall, at $y=-h$; cf. Fig. 1) and $U_{c}$ is the mean velocity at the channel center, $y=0$. In other words, $u_{\tau}$ is the only velocity scale and $h$ the only length scale for this problem (scaling with $u_{\tau}$ and $h$ or with $\mathrm{d}(P / \rho) / \mathrm{d} x$ and $h$ is actually equivalent), and away from the walls $\mathcal{F}(y / h)$ is independent of Reynolds number. Simple algebra on the velocity gradient $\mathrm{d} U / \mathrm{d} y$ reveals that (2) and (3) are compatible, so that (2) amounts to an overlap argument between (1) and (3), exactly in the way the logarithmic law follows from an overlap between the law of the wall $U^{+}=\mathcal{F}\left(y^{+}\right)$and the defect law (3). The degree to which the present results satisfy (3) will also be of great interest since the present flow, by definition, has a pressure gradient of order one, and the FPG wall is of course a candidate for universal behavior, of the common type, extending into the core region.

\section{Approach and Problem Definition}

Results for $\operatorname{Re}=3000,6000$ and 12000 are considered below. The parameters used for the various cases are tabulated in Tables 1 and 2.

To put this study on as firm a foundation as possible, we apply three separate codes - one finite-difference (Code A; PBO11), one mixed-discretization (Code B; Luchini \& Quadrio [7]) and one fully spectral (Code C; Johnstone et al. [5]) - to the same flow conditions, with $\operatorname{Re}=3000$ and $\mathrm{d}(P / \rho) / \mathrm{d} x=-0.0044 U_{w}^{2} / h$, in the same domain, assuming periodic conditions in the streamwise $x$ and spanwise $z$ directions, with periods $\Lambda_{x}=4 \pi h$ and $\Lambda_{z}=2 \pi h$. These three runs are respectively denoted Case A3000, B3000 and C3000a. Streamwise-velocity flow visualizations (shown later in Figs. 6 and 7) suggest that the present domain size is sufficient, but spanwise spectra (not shown) from a $\operatorname{Re}=3000$ flow have a significant content at a wavelength of $\pi h$ (that is, half of the simulation period) with essentially no $x$-dependence. This is somewhat confirmed by Fig. $7 \mathrm{~b}$ and $\mathrm{c}$, raising the possibility that further increases of $\Lambda_{z}$ are required to reach the ultimate level of accuracy, particularly in the core region. Motivated by this observation, Case C3000b was run, which is a larger-domain version of Case C3000a, with $\Lambda_{x} / h=6 \pi h$ and $\Lambda_{z}=4 \pi h$ (i.e., 1.5 and 2 times larger, respectively); its spatial (and temporal) resolution is identical (see Table 2).

(a)

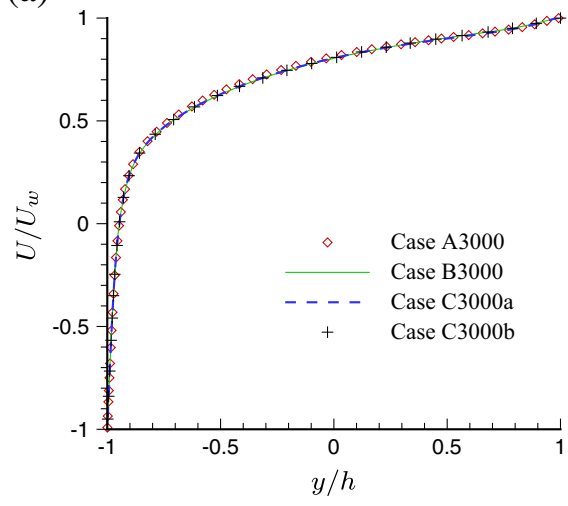

(b)

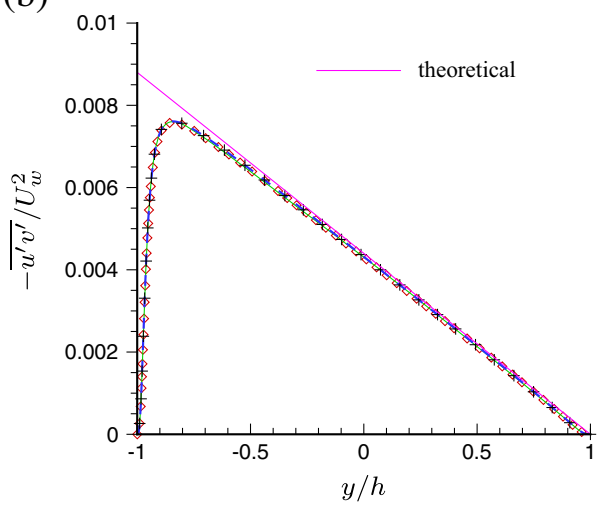

Fig. 1 Profiles of (a) mean velocity and (b) Reynolds shear stress for Re $=3000$ from three DNS codes. Straight line in (b) indicates total-stress profile defined by pressure gradient 
Table 1 Case parameters

\begin{tabular}{llllllll}
\hline Case & Code & $\operatorname{Re}$ & $\left(-\frac{\mathrm{d} P}{\mathrm{~d} x}\right)\left(\frac{h}{\rho U_{w}^{2}}\right)$ & $T_{A} U_{w} / h$ & $\xi$ & $\tau_{w, \mathrm{APG}} / \rho u_{\tau}^{2}$ & $u_{p} / u_{\tau}$ \\
\hline $\mathrm{A} 3000$ & $\mathrm{~A}$ & 3000 & 0.0044000 & 2000 & 0.014 & $-6.6 \times 10^{-4}$ & 0.1212 \\
$\mathrm{~B} 3000$ & $\mathrm{~B}$ & 3000 & 0.0044000 & 2000 & 0.053 & $-2.8 \times 10^{-4}$ & 0.1213 \\
$\mathrm{C} 3000 \mathrm{a}$ & $\mathrm{C}$ & 3000 & 0.0044000 & 1867 & 0.003 & $-1.2 \times 10^{-3}$ & 0.1211 \\
$\mathrm{C} 3000 \mathrm{~b}$ & $\mathrm{C}$ & 3000 & 0.0044000 & 688 & 0.006 & $-2.6 \times 10^{-3}$ & 0.1211 \\
$\mathrm{~A} 6000^{\dagger}$ & $\mathrm{A}$ & 6000 & $0.0037836^{\dagger}$ & 2000 & 0.016 & $0^{\dagger}$ & 0.0986 \\
$\mathrm{C} 6000 \mathrm{a}$ & $\mathrm{C}$ & 6000 & 0.0038240 & 1906 & 0.003 & $+3.4 \times 10^{-3}$ & 0.0982 \\
$\mathrm{C} 6000 \mathrm{~b}$ & $\mathrm{C}$ & 6000 & 0.0039440 & 908 & 0.006 & $-1.5 \times 10^{-3}$ & 0.0980 \\
$\mathrm{C} 12000 \mathrm{a}$ & $\mathrm{C}$ & 12000 & 0.0032894 & 1779 & 0.007 & $+6.5 \times 10^{-3}$ & 0.0796 \\
$\mathrm{C} 12000 \mathrm{~b}$ & $\mathrm{C}$ & 12000 & 0.0034514 & 1004 & 0.006 & $+1.6 \times 10^{-3}$ & 0.0794 \\
\hline
\end{tabular}

Details of DNS algorithms given in Pirozzoli et al. [9] (Code A; see also Orlandi [8]), Luchini \& Quadrio [7] (Code B) and Johnstone et al. [5] (Code C). The momentum-imbalance parameter $\xi$ (which would equal zero for perfect balance) is defined in the text. The statistics were gathered by averaging over $x-z$ planes at discrete times over the period $T_{A}$. Note that case names used here do not always correspond to the parameters defined in Coleman et al. [3]

${ }^{\dagger}$ Case A6000 uses a spatially uniform time-dependent pressure gradient adjusted such that the APG-wall shear $\tau_{w, \text { APG }}$ remains essentially zero (i.e., the one-sided three-point estimate of this derivative is machine zero on a 64-bit machine). Value of $\mathrm{d} P / \mathrm{d} x$ shown is the time average

This flow field also contains large-scale longitudinal 'ridges,' suggesting that the CP0 flow poses a challenge in terms of periodic domain size, although much milder than it is for pure Couette flow. It is difficult to ascertain when the solution has truly erased any memory of its initial condition, especially regarding the larger structures, with their long time scales. The very good agreement of the statistics from these two cases shown below indicates that the overall conclusions drawn from the present DNS results are not adversely affected by the domain size.

Table 2 Numerical parameters

\begin{tabular}{lllllllll}
\hline Case & $\Lambda_{x} / h$ & $\Lambda_{z} / h$ & $N_{x}$ & $\Delta x^{+}$ & $N_{y}$ & $y_{10}^{+}$ & $N_{z}$ & $\Delta z^{+}$ \\
\hline A3000 & $4 \pi$ & $2 \pi$ & 512 & 6.9 & 192 & 2.4 & 384 & 4.6 \\
B3000 & $4 \pi$ & $2 \pi$ & 512 & 6.9 & 151 & 10.4 & 512 & 3.5 \\
C3000a & $4 \pi$ & $2 \pi$ & 576 & 6.1 & 257 & 1.7 & 432 & 4.1 \\
C3000b & $6 \pi$ & $4 \pi$ & 864 & 6.1 & 257 & 1.7 & 864 & 4.1 \\
A6000 & $4 \pi$ & $2 \pi$ & 1024 & 6.4 & 384 & 1.8 & 768 & 4.3 \\
C6000a & $4 \pi$ & $2 \pi$ & 576 & 11.5 & 257 & 3.2 & 864 & 3.8 \\
C6000b & $4 \pi$ & $2 \pi$ & 576 & 11.6 & 257 & 3.2 & 864 & 3.9 \\
C12000a & $4 \pi$ & $2 \pi$ & 1152 & 10.7 & 385 & 2.6 & 1536 & 4.0 \\
C12000b & $4 \pi$ & $2 \pi$ & 1152 & 10.9 & 257 & 6.1 & 1536 & 4.1 \\
\hline
\end{tabular}

Resolution given in terms of equivalent streamwise $N_{x}$, wall-normal $N_{y}$ and spanwise $N_{z}$ grid points (with de-aliasing in $x$ and $z$ for Codes B and C, such that the number of expansion coefficients is $2 / 3$ the number of grid points in these directions), and the resulting FPG-wall units $\Delta x^{+}=\Delta x u_{\tau} / v, y_{10}^{+}=\left(h+y_{10}\right) u_{\tau} / v$ and $\Delta z^{+}=\Delta z u_{\tau} / v$, where $\Delta x=\Lambda_{z} / N_{x}, \Delta z=\Lambda_{z} / N_{z}, \Lambda_{x}$ is the streamwise, and $\Lambda_{z}$ the spanwise, domain size and $y_{10}$ is the $y$-coordinate of the 10th grid point from the bottom of the domain (9th grid point off the wall) 
The pressure-gradient/wall-velocity combination used for the $\mathrm{Re}=3000$ runs yields negligible skin friction on the APG wall (Fig. 1), in that the stress on that wall is at most of the order of $0.25 \%$ of the FPG-wall value (Table 1 ). The number of grid points used for each case, and the corresponding spatial resolution in FPG-side wall units, are listed in Table 2. The APG wall region is far calmer (see Fig. 7a) and therefore less demanding of resolution in space and time. Therefore, we believe it is very well resolved. For nearly all the runs, the resolution in all three directions is well within the bounds needed for a spectral discretization (such as that used by Codes B and C) to produce accurate first- and second-order statistics in a turbulent boundary layer with finite skin friction (i.e., $\Delta x^{+} \leq 12, \Delta z^{+} \leq 4, y_{10}^{+} \leq 6$; cf. Spalart et al. [11] and see Table 2 caption). These values are expected to also be reasonable for the finite-difference code/directions. More direct confirmation of the appropriateness of the resolution, especially on the APG side, will be inferred by comparing results from the different codes, as well as recent channel results. Codes $\mathrm{A}$ and $\mathrm{C}$ are also applied at $\mathrm{Re}=6000$, for Cases A6000, C6000a and $\mathrm{C} 6000 \mathrm{~b}$, with pressure gradients tuned to yield $\mathrm{d} U / \mathrm{d} y \approx 0$ (or in the former case, $\mathrm{d} U / \mathrm{d} y \equiv 0)$ on the APG wall; the latter two spectral-code runs differ from each other only in their pressure gradients, which produce slightly different mean APG-wall shear $\mathrm{d} U / \mathrm{d} y_{\mathrm{APG}}$, of opposite sign but very small (Table 1 ). The two $\mathrm{Re}=12000$ cases are also identical apart from their $\mathrm{d} P / \mathrm{d} x$ values, which again yield slightly different $\mathrm{d} U / \mathrm{d} y_{\mathrm{APG}}$, of about +0.7 and $+0.2 \%$ of their FPG-wall shears. These dual- $\mathrm{d} P / \mathrm{d} x$ results at $\mathrm{Re}=6000$ and 12000 will be used to estimate the $U$ profiles corresponding to the $\mathrm{d} U / \mathrm{d} y \equiv 0$ idealization at each Re, by forming an appropriate linear combination of the two profiles (see below).

Turbulent initial conditions were obtained either by applying random velocity disturbances across the domain, or from a mature field from another case at lower Reynolds number. For example, for Case C12000a, an early Case C6000a field was projected onto a finer grid (Table 2) and the wall velocities adjusted to minimize the changes to net streamwise momentum. This involved invoking (3) in the core region while maintaining the same $\mathrm{d}(P / \rho) / \mathrm{d} x$ (with respect to $U_{w}$ at $\operatorname{Re}=6000$ ), and thereby roughly the same $u_{\tau}$, employed for Case A6000. (The first $\mathrm{Re}=6000$ runs with code $\mathrm{C}$ also employed the mean pressure gradient obtained from Case A6000. However, the small but finite positive residual $\mathrm{d} U / \mathrm{d} y_{\mathrm{APG}}$, of the order of $0.003 \mathrm{~d} U / \mathrm{d} y_{\mathrm{FPG}}$ - presumably due to differences of statistical and numerical convergence between the two runs and the codes that produced them and our observation that these small deviations from zero of the APG-wall stress have a profound effect on the square-root scaling (2), led to the decision to increase $\mathrm{d}(P / \rho) / \mathrm{d} x$ slightly, relative to Case A6000, for Cases C6000a and C6000b.)

The global/wall-to-wall momentum balance, $\left(\tau_{\mathrm{FPG}}-\tau_{\mathrm{APG}}\right) / 2 h[\mathrm{~d} P / \mathrm{d} x]$, lies between 0.998 and 1.006 for all the cases shown in Table 1. (Recall the ideal value is unity.) A more discriminating measure of the mean-momentum imbalance, reflecting the quality of the statistics by its nearness to zero, is quantified in terms of the root-mean-squared difference between the two right-hand-side terms of the mean streamwise RANS equation. This quantity is defined as

$$
\xi=\left[\frac{1}{2 h} \int_{-h}^{+h}\left[\frac{\mathrm{d}(\tau / \rho)}{\mathrm{d} y}-\frac{\mathrm{d}(P / \rho)}{\mathrm{d} x}\right]^{2} \mathrm{~d} y\right]^{\frac{1}{2}} / \frac{-\mathrm{d}(P / \rho)}{\mathrm{d} x}
$$

where $\tau / \rho=v \mathrm{~d} U / \mathrm{d} y-u^{\prime} v^{\prime} ;$ it is included in Table 1 . 


\section{Results}

\subsection{Mean Velocity}

The $\mathrm{Re}=3000$ results from the three codes are presented in Fig. 1. The close agreement points to the accuracy and reliability of the DNS data, at least for these domain sizes, and to the adequacy of the spatial resolution used in each case (which implies that that used for A3000 and C3000a, especially in the wall-normal direction, is much finer than is required to capture faithfully the $U$ and $-\overline{u^{\prime} v^{\prime}}$ profiles). The APG-side skin friction is also a very discriminating measure of the agreement between codes.

A comparison across all three Re values is shown in Fig. 2. Figure 2a normalizes $U(y)$ with $U_{w}$, and the effect of Reynolds number is clear. Contrast with Fig. 1b, a test of the outer-layer scaling (3), with respect to the friction velocity $u_{\tau}$; the striking collapse across the core of the flow supports Townsend's assumption that $u_{\tau}$ is the relevant outer-layer velocity scale, and that Reynolds-number effects are limited to thin regions near the walls.

(a)

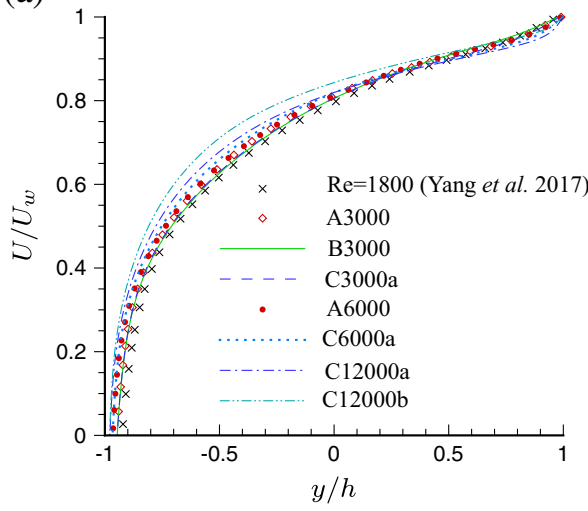

(b)

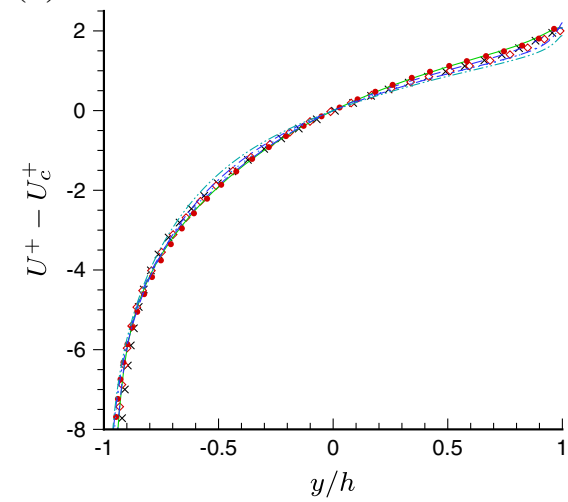

(c)

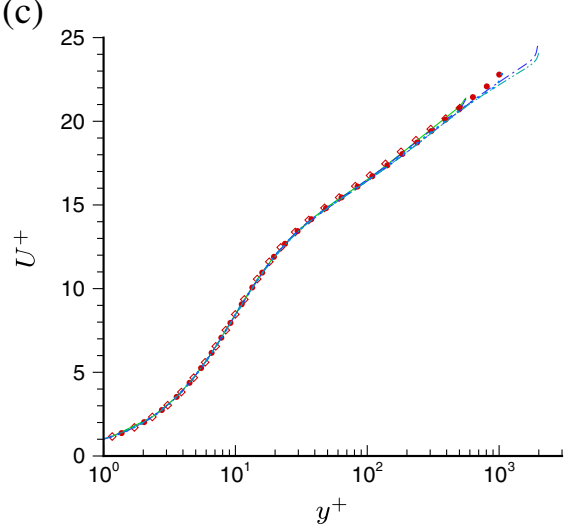

Fig. 2 Mean-velocity profiles scaled with respect to (a) $U_{w}$ and (b,c) $u_{\tau}$, in terms of (b) outer $y / h$ coordinate (relative to centerline velocity $\left.U_{c}^{+}=U(0) / u_{\tau}\right)$ and (c) inner $y^{+}$coordinate, where $y^{+}=(y+h) u_{\tau} / \nu$. For $\mathrm{Re}=3000,6000$ and 12000 , respectively, $u_{\tau} / U_{w}=0.0937$ (A3000,B3000,C3000a), 0.0870 (A6000), 0.0877 (C6000a) and $0.0816(\mathrm{C} 12000 \mathrm{a}), 0.0832(\mathrm{C} 12000 \mathrm{~b})$ such that $h^{+}=281$ (A3000,B3000,C3000a), 522 (A6000), 526 (C6000a) and 979 (C12000a), 998 (C12000b) 
The inner scaling for the FPG wall is shown in Fig. 2c for the mean velocity and in Fig. 4a for the Reynolds stresses. The behavior is highly reminiscent of that seen in pure planePoiseuille flow. We note the approach toward zero of the pressure gradient with Reynolds number, implied by the reduction in slope of the shear stress profile in Fig. 4a (i.e., that the pressure gradient in wall units $p^{+}$goes to zero as Re increases). The logarithmic $U^{+}$profile in Fig. 2c reveals the manner in which this flow essentially doubles the thickness of the inertial sublayer contained between the walls, compared to the plane-channel counterpart with the same value for $h u_{\tau} / v$.

A test of conjectures (I) and (II) (i.e., of Eqs. 1 and 2) is provided by the $u_{p}, v$ scaling shown in Fig. 3, which contains the $\mathrm{Re}=3000$ profiles from Fig. 1, as well as the results from the $\operatorname{Re}=6000$ and 12000 runs. Also included are the recent $\operatorname{Re}=1800$ CP0 DNS data of Yang et al. [14]. The code-C profiles for $\operatorname{Re}=6000$ and 12000 are $\mathrm{d} U / \mathrm{d} y_{\mathrm{APG}}=0$ approximations obtained from linear combinations of, respectively, Cases C6000a \& C6000b and Cases C12000a \& C12000b, with weighting coefficients defined such that the resultant satisfies $U=U_{w}$ and $\mathrm{d} U / \mathrm{d} y=0$ at $y=+h$. This strategy was prompted by the surprisingly large effect of very small values of $\mathrm{d} U / \mathrm{d} y$ on the APG wall values even as small as those recorded in Table 1 for the $\mathrm{Re}=6000$ and 12000 runs with Code $\mathrm{C}$ - have on the slope $B$ and especially additive constant $C$ of the $U^{-}=U^{-}\left(\sqrt{y^{-}}\right)$ scaling. The contributions of Reynolds number, statistical convergence and type of (uniform) pressure gradient (time-dependent versus constant) to this sensitivity is a subject for future study. For now, we note that $u_{p} / u_{\tau}$ decreases with increasing Re (Table 1).

Although differences of the order of one $U^{-}$unit remain, we consider this set of results to be strongly in favor of both of Stratford's conjectures, which is quite a positive outcome. Visual fits suggest the approximate range [2.25, 2.50] for $B$ and $C \approx-2.2$ (see straightline interpolants in Fig. 3). These are considerably tighter than the ranges which were in place before the present study, even limited to CP0 DNS. The square-root law appears to smoothly extend to higher $y^{-}$as the Reynolds number increases, just as the log law does. We effectively observe agreement between four independent codes and researchers.
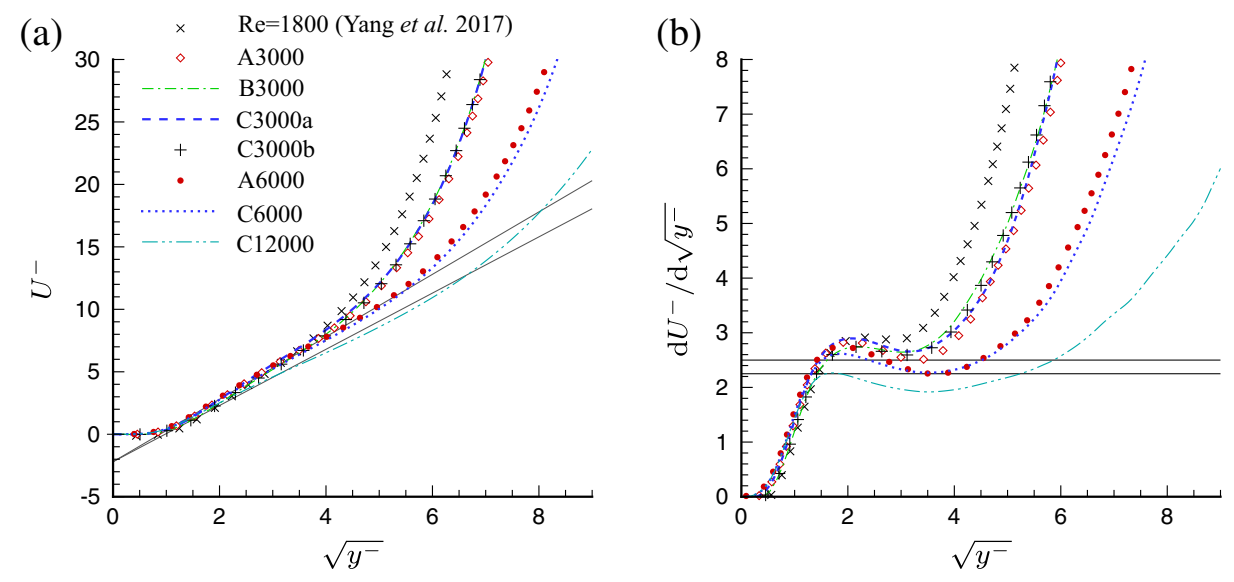

Fig. 3 Mean-velocity profiles in Stratford units. For Re = 1800 (Yang et al. [14]), 3000, 6000 and 12000 , respectively, $u_{p} / U_{w}=0.01405,0.01136$ (A3000,B3000,C3000a), 0.00858 (A6000), 0.00867 (C6000) and 0.00664 (C12000), such that $h^{-}=25.3,34.1$ (A3000,B3000,C3000a), 51.45 (A6000), 52.0 (C6000) and 79.6 (C12000). C6000 and C12000 profiles are linear combination of C6000a \& C6000b, and C12000a \& $\mathrm{C} 12000 \mathrm{~b}$ results, respectively. Straight lines correspond to Eq. 2 with $B=2.25$ and 2.50 , both with $C=-2.2$ 
A theoretical concern is that the square-root argument depends on large values of $y^{-}$, just like the logarithmic law appears only for $y^{+} \gg 1$ (in practice, at least 50, but recent findings suggest a value of several hundred), and at first sight the $y^{-}$range (roughly, from 4 to 20 in Fig. 3) may not yet be large enough. In other words, the appearance of a square-root layer could appear premature.

A counter-argument is the observation that the ratio of the total-to-viscous shear stress within the $y_{w}^{1 / 2}$ regime (where Eq. 2 is valid) is given by $2\left(y^{-}\right)^{3 / 2} / B$; assuming $B=\mathcal{O}(2)$ (cf. PBO11; SC15), this implies that even at the bottom of the range of $U \sim y_{w}^{1 / 2}$ behavior shown in Fig. 3a, near $y^{-}=4$, the turbulent stress is already an order of magnitude larger than the viscous component. At the upper limit of the validity of Eq. 2 found for $\operatorname{Re}=6000$, $y^{-} \approx 20$, the turbulent/viscous stress ratio is approaching 100. Based on this reasoning $\left(y^{-}\right)^{3 / 2}$, rather than $y^{-}$or $\left(y^{-}\right)^{1 / 2}$, is the quantity that needs to be 'large' compared with unity for the argument that viscosity has no influence locally to be valid. The equivalent ratio in a $\log$ law is $\kappa y^{+}$, which is only about 40 at $y^{+}=100$, where the log law may be reasonably expected to begin. We note that $\left(h^{-}\right)^{3 / 2}=\left(h u_{p} / v\right)^{3 / 2} \approx 200$ for the $\operatorname{Re}=3000$ flow, and increases to $\approx 370$ at $\operatorname{Re}=6000$ and $\approx 700$ at $\operatorname{Re}=12000$. (Compare the corresponding increase in the channel half-height in viscous units from $h^{+}=h u_{\tau} / v$ of about 280 to 520 to 990 .) These considerations support the relevance of the present DNS as a means to address the validity of Eqs. 1 and 2, in the sense that the present Reynolds number range is not grossly insufficient.

\subsection{Reynolds Stresses}

The Reynolds stresses and their dependence on Re are illustrated in Fig. 4. (Henceforth, only Code $\mathrm{C}$ results will be considered.) We have already mentioned the similarity of the FPGwall scaled stresses (Fig. 4a) to those observed in the plane channel; the significant variation

(a)

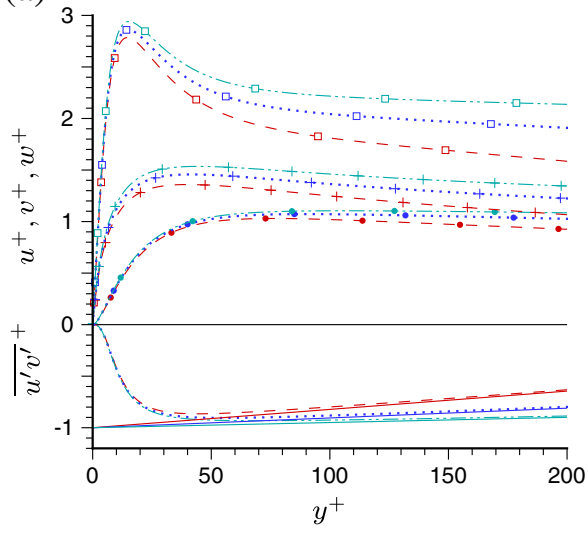

(b) $\quad \ldots+\cdots$

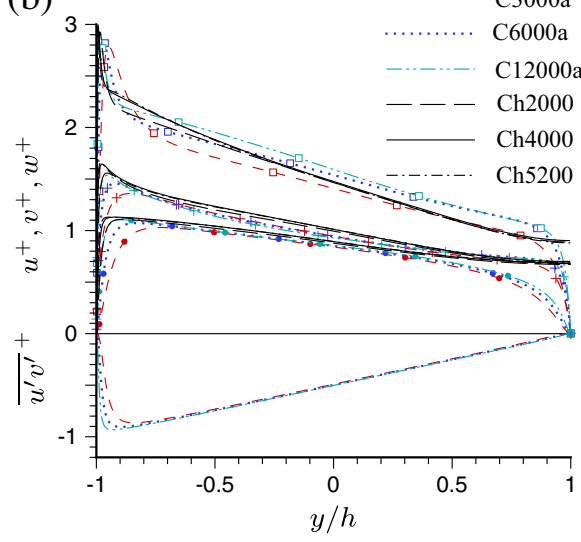

Fig. 4 FPG-wall scaling of Reynolds-shear-stress and turbulence-intensity profiles: $\square, u^{+}=\sqrt{\overline{u^{\prime} u^{\prime}}} / u_{\tau}$; , $v^{+}=\sqrt{\overline{\overline{v^{\prime} v^{\prime}}}} / u_{\tau} ;+, w^{+}=\sqrt{\overline{\overline{w^{\prime} w^{\prime}}}} / u_{\tau} ;$ no symbol, $\overline{u^{\prime} v^{\prime}}=\overline{\overline{u^{\prime} v^{\prime}}} / u_{\tau}^{2}$. Slope of solid lines in (a) corresponds to $-p^{+}=-[\mathrm{d}(P / \rho) / \mathrm{d} x]\left[v / u_{\tau}^{3}\right]$. Cases Ch2000, Ch4000 and Ch5200 in (b) are from plane-channel $\left(U_{w} \equiv\right.$ 0) DNS of, respectively, Hoyas and Jiménez [4] $\left(\operatorname{Re}_{\tau}=2003\right)$, Bernardini et al. [1] $\left(\operatorname{Re}_{\tau}=4079\right)$ and Lee and Moser [6] $\left(\operatorname{Re}_{\tau}=5186\right)$, where for illustration purposes the wall-to-centerline half-domain from that flow has been mapped to the full bottom-to-top-wall domain here 


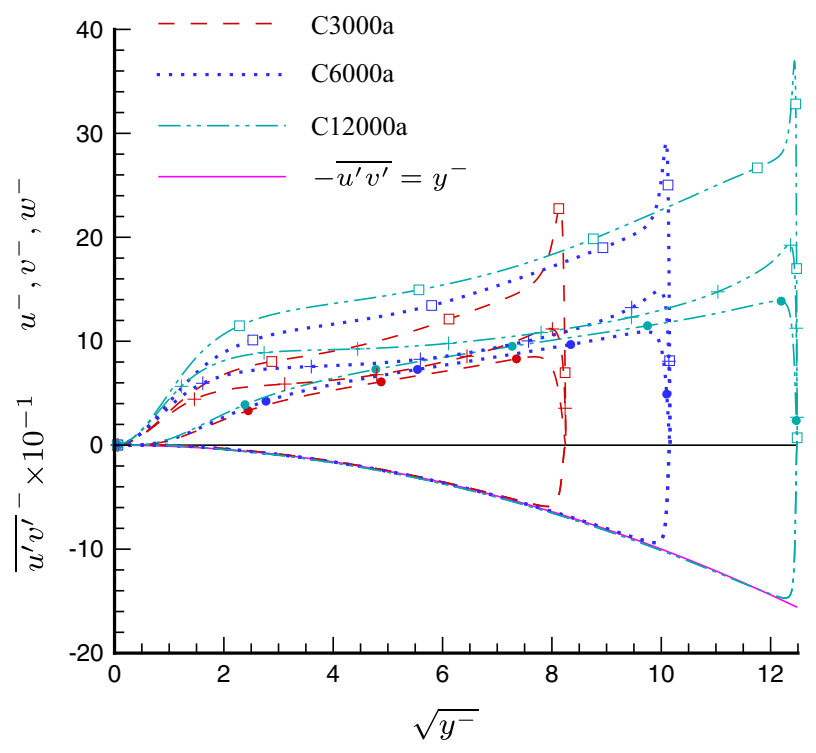

Fig. 5 APG-wall scaling of Reynolds-shear-stress and turbulence-intensity profiles with respect to $y^{-}=$ $(h-y) u_{p} / v: \square, u^{-}=\sqrt{\overline{\overline{u^{\prime} u^{\prime}}}} / u_{p} ; \bullet, v^{-}=\sqrt{\overline{v^{\prime} v^{\prime}}} / u_{p} ;+, w^{-}=\sqrt{\overline{\overline{w^{\prime} w^{\prime}}}} / u_{p} ;$ no symbol, $\overline{u^{\prime} v^{\prime}}=\overline{\overline{u^{\prime} v^{\prime}}} / u_{p}^{2}$. Solid line traces theoretical variation, $-\overline{u^{\prime} v^{\prime}}=y^{-}$

with Reynolds number of all three normal stresses measured in units of $u_{\tau}$ versus $y^{+}$has been noted in many studies (without, however, any certainty on whether this is a 'lowReynolds-number effect' that will ultimately saturate). The collapse and linear behavior in the core-layer variable $y / h$ of the $-\overline{u^{\prime} v^{\prime}}$ shear stresses across the core is required in this scaling (Fig. 4b). The wall-normal $v^{+}$and spanwise $w^{+}$components also tend to collapse in the core region, as they do in pure Poiseuille flow (when their 'folded' wall-to-centerline half domains are expanded to fill the full bottom-to-top wall domain of the present CP0 flow, as is done in Fig. $4 \mathrm{~b}$ for the $u_{\tau} h / v \approx 2000,4000$ and 5200 DNS of Hoyas and Jiménez [4], Bernardini et al. [1] and Lee and Moser [6], respectively.) The behavior of the streamwise component $\sqrt{\overline{u^{\prime} u^{\prime}}}$, on the other hand, differs from that of the Poiseuille case, for

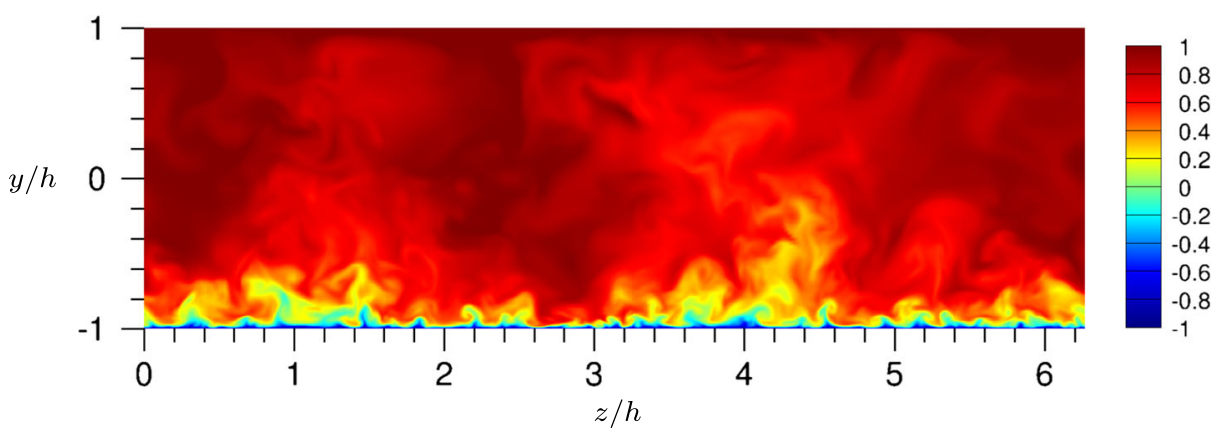

Fig. 6 Instantaneous contours of streamwise velocity $u$ in cross-stream-vertical plane from CP0 DNS $(\operatorname{Re}=$ 6000) 
(a)
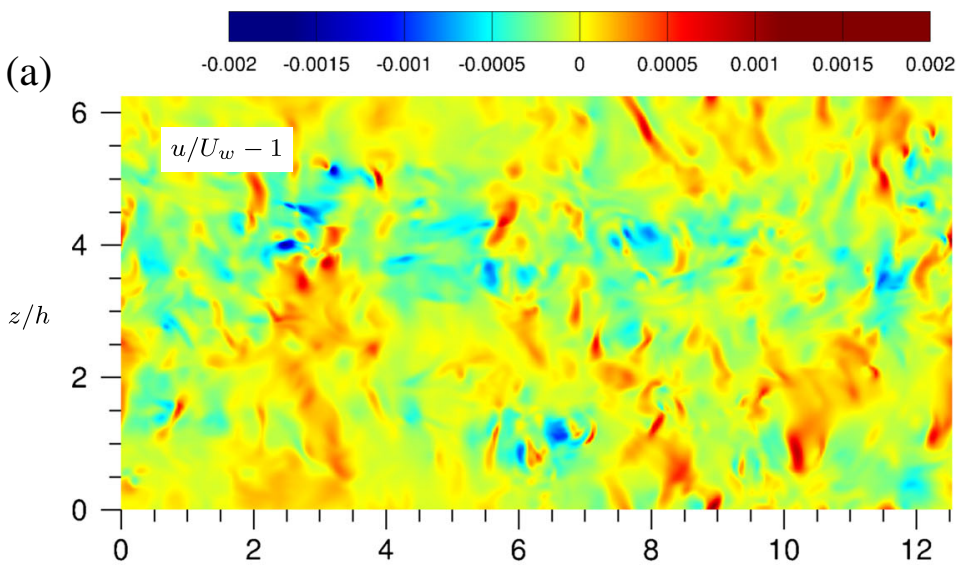

(b)

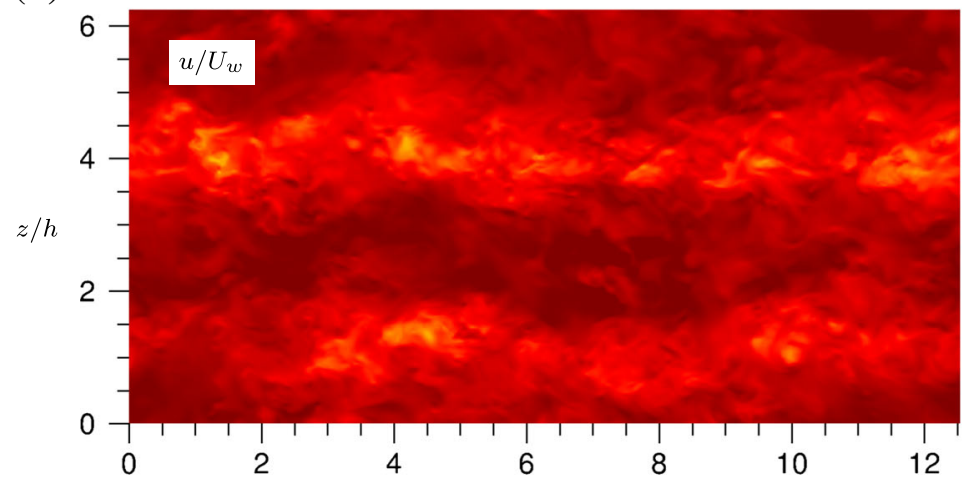

(c)
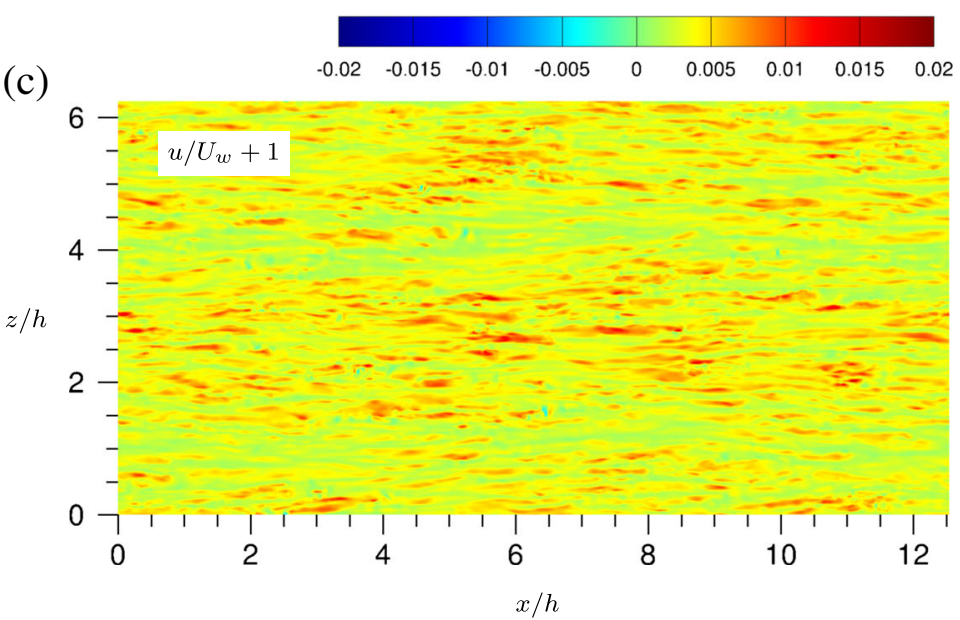

Fig. 7 Instantaneous contours of streamwise velocity $u$ in wall-parallel planes at (a) $y=+0.9999 h$ (near APG wall), b $y=0$ (centerline), and $(c) y=-0.9999 h$ (near FPG wall), from CP0 DNS $(\operatorname{Re}=6000)$ 
which only in the inner layer and bottom of the outer layer does it become an increasingly large fraction of $u_{\tau}$ as Reynolds number increases. Here the profiles remain linear across the bulk of the flow and the step with Re appears almost uniform in space, and identical for the two Reynolds-number doublings, so that there is little indication that the rise will saturate rapidly at even higher Re.

The Reynolds stresses in the zero-stress-wall scaling are presented in Fig. 5. The $-\overline{u^{\prime} v^{\prime}}$ component agrees well with the theoretical profile, $-\overline{u^{\prime} v^{\prime}}-y^{-}$, for each of the three Reynolds numbers, as required by the momentum balance; the viscous stress is not visible in these coordinates except near the opposite wall. This agreement also suggests the success of the pressure-gradient-and-wall-velocity combinations used here to establish the CP0 conditions. As is the case for the FPG-side fluctuations in the $u_{\tau}$ scaling, the normal-stress components in the $u_{p}$ scaling fail to exhibit Reynolds-number independence, with the deviation for the streamwise component being especially pronounced. Thus, the turbulence as a whole definitely does not scale with only $u_{p}$ and $y^{-}$even if the velocity and shear stress do.

\subsection{Flow Visualizations}

The instantaneous streamwise velocity contours in a $y-z$ plane shown in Fig. 6 suggest the turbulence associated with the FPG wall affects the flow all the way to the APG side. Eruptions from the intense FPG region reach fairly close to the quiescent APG layer. See also the contours in wall-parallel $x-z$ planes in Fig. 7. These contours also reveal the presence of coherent large-scale roll-type structures associated with the second spanwise mode; this is particullary evident in the $y=0$ centerline plane (Fig. 7b). However, neither of these wall-to-wall phenomena appear to defeat the Stratford argument, or the universality implied by the present DNS data. This claim is supported in Fig. 3 by the good agreement between the $U^{-}$profiles from Cases C3000a and 3000b, with their respectively smaller $\left(\Lambda_{x} / h=4 \pi, \Lambda_{z} / h=2 \pi\right)$ and larger $\left(\Lambda_{x} / h=6 \pi, \Lambda_{z} / h=4 \pi\right)$ domains.

\section{Summary and Future Work}

Our joint study of the Couette-Poiseuille flow with zero skin friction on one wall by Direct Numerical Simulation reached its objectives quite well. Away from the zero-skin-friction wall the mean velocity and Reynolds shear stress are dominated by the friction velocity $u_{\tau}$ and the channel half-height $h$, as it is in simple Poiseuille flow. The streamwise Reynolds stress is again not universal with any normalization we attempted.

The confirmation of the Stratford square-root law, even if it is restricted to small regions or a very particular flow, is quite satisfying to the theoretician, in that a simple analytical form similar to the Kolmogorov inertial range or the logarithmic law was predicted purely by reasoning on the dominance of a parameter (here, the pressure gradient), and the limited role of viscosity.

In the future, higher Reynolds numbers may refine the estimates of the Stratford constants. (In light of the reduction with increasing Reynolds number of $u_{p} / u_{\tau}$ (cf. Table 1), those who perform $\mathrm{CPO}$ simulations at higher Re are reminded to attend to the sensitivity of the Stratford scaling to even very weak nonzero skin friction on the APG wall.) Further variation of the periodic domain size is in order, but is not expected to alter the general conclusions. An experiment on the CP0 flow would be of great interest, provided accurate measurements are possible close enough to a sliding belt, and a large enough domain eliminates end effects in both $x$ and $z$ directions. 
The now better-understood CP0 flow also becomes a candidate to be a standard calibration case for turbulence models. We have determined that models of the classical type will naturally satisfy Stratford scaling and produce square-root layers, but for instance the Spalart-Allmaras model does not give very accurate values for the $B$ and $C$ constants.

Looking beyond the ideal CP0 situation, it will be of great interest to explore other flows with zero skin friction. For instance, a separating boundary layer may or may not sustain zero skin friction over a large enough region for the scaling to establish itself. The mean wall-normal velocity $V$, or the strain $\partial V / \partial y$, may also conflict with the pressure gradient as a dominating influence. In our other studies we have DNS in which the skin friction crosses zero, but not of Stratford's 'continuously separating' flow, which will be quite challenging because the boundary-layer thickness increases so rapidly. The current indications are that the square-root law indeed applies at both zero crossings, and with the same constants found here.

Acknowledgements This research was sponsored by NASA's Transformational Tools and Technologies (TTT) Project of the Transformative Aeronautics Concepts Program under the Aeronautics Research Mission Directorate. Computations were performed on resources provided by the NASA Advanced Supercomputing (NAS) Division, while the results of the Sapienza unit have been achieved using the PRACE Research Infrastructure resource FERMI based at CINECA, Casalecchio di Reno, Italy. We are grateful to Dr. Yang of NTNU for providing her DNS data.

Conflict of interests The authors declare that they have no conflict of interest.

\section{References}

1. Bernardini, M., Pirozzoli, S., Orlandi, P.: Velocity statistics in turbulent channel flow up to $R e_{\tau}=4000$. J. Fluid Mech. 742, 171-191 (2014)

2. Coleman, G.N., Spalart, P.R.: Direct numerical simulation of turbulent Couette-Poiseuille flow with zero skin friction. In: Proceedings of 15th European Turbulence Conference, pp. 25-28. Delft (2015). referred to herein as CS15

3. Coleman, G.N., Pirozzoli, S., Quadric, M., Spalart, P.R.: Direct numerical simulation of a wallbounded flow with zero skin friction. In: Proceedings of 11th International ERCOFTAC Symposium on Engineering Turbulence Modelling and Measuremens, pp. 21-23, Palermo (2016)

4. Hoyas, S., Jiménez, J.: Scaling of the velocity fluctuations in turbulent channels up to $\operatorname{Re}_{\tau}=2003$. Phys. Fluids 18, 011702 (2006)

5. Johnstone, R., Coleman, G.N., Spalart, P.R.: The resilience of the logarithmic law to pressure gradients: evidence from direct numerical simulation. J. Fluid Mech. 643, 163-175 (2010)

6. Lee, M., Moser, R.D.: Direct numerical simulation of turbulent channel flow up to $R e_{\tau} 5200$. J. Fluid Mech. 774, 395-415 (2015)

7. Luchini, P., Quadrio, M.: A low-cost parallel implementation of direct numerical simulation of wall turbulence. J. Comp. Phys. 211(2), 551-571 (2006)

8. Orlandi, P.: Fluid flow phenomena. A Numerical Toolkit, Kluwer (2000)

9. Pirozzoli, S., Bernardini, M., Orlandi, P.: Large-scale motions and inner/outer layer interactions in turbulent Couette-Poiseuille flows. J. Fluid Mech. 680, 534-563 (2011). referred to herein as PBO11

10. Schlichting, H., Gersten, K.: Boundary layer theory. 8th edn, Springer (2000)

11. Spalart, P.R., Coleman, G.N., Johnstone, R.: Retraction: 'Direct numerical simulation of the Edman layer: A step in Reynolds number, and cautious support for a log law with a shifted origin'. Phys. Fluids 21, 109901 (2009)

12. Stratford, B.: The prediction of separation of the turbulent boundary layer. J. Fluid Mech. 5, 1-16 (1959)

13. Townsend, A.A.: The structure of turbulent shear flow. 2nd edn, Cambridge (1976)

14. Yang, K., Zhao, L., Andersson, H.I.: Turbulent Couette-Poiseuille flow with zero wall shear. Int. J. Heat Fluid Flow 63, 14-27 (2017) 